\title{
The Influence of Previous Arthroscopic Treatment on Subsequent Total Knee Arthroplasty: the Comparison Between Bilateral Knees of the Same Patient
}

\author{
Kui-shuai XU \\ the Affiliated Hospital of Qingdao University \\ Liang ZHANG \\ Affiliated Hospital of Qingdao University \\ Rui SHEN \\ the Affiliated Hospital of Qingdao University \\ Cai-lin WANG \\ Wenzhou Medical University \\ Tian-yu LI \\ the Affiliated Hospital of Qingdao University
}

\section{Xia ZHAO}

the Affiliated Hospital of Qingdao University

Teng-bo YU ( $\nabla$ ytb8912@hotmail.com )

the Affiliated Hospital of Qingdao University https://orcid.org/0000-0003-1134-0134

\section{Research article}

Keywords: Influence, Arthroscopy, Total knee arthroplasty, same patient

Posted Date: October 30th, 2020

DOI: https://doi.org/10.21203/rs.3.rs-97253/v1

License: (c) (1) This work is licensed under a Creative Commons Attribution 4.0 International License. Read Full License

Version of Record: A version of this preprint was published on January 29th, 2021. See the published version at https://doi.org/10.1186/s12891-021-04003-3. 


\section{Abstract}

Purpose To explore whether previous arthroscopic knee surgery affects future total knee arthroplasty results or not.

Methods A total of 56 patients with the previous arthroscopic treatment on one knee underwent subsequent bilateral total knee arthroplasty in our hospital from September 2012 to July 2020. Data on each patient were collected in regards to changes in postoperative clinical and functional scores, various other scores, as well as postoperative functional recovery and complications. We defined the knees with a previous arthroscopic history as group A, and the counter side as group B.The Knee Society clinical score, functional scores, ROM, FJS, VAS scores were assessed before and after surgery. Using the KolmogorovSmirnov Test to test the normality of continuous variables, and the chi-square test to compare the rate of reoperation and complications between two groups. For all statistical comparisons, $\mathrm{P}<0.05$ was considered significant.

Results There were no statistically significance differences found in postoperative Knee Society clinical scores and functional scores between group A and group B, as well as in ROM, FJS, VAS scores and local complications.

Conclusion There were no statistically significant differences found in postoperative functional recovery and complications in patients, who underwent total knee arthroplasty with previous knee arthroscopy.

\section{Background}

The global burden of disease 2010 study showed that the global morbidity of symptomatic knee osteoarthritis was estimated to be $3.8 \%$, and that the morbidity increases with age, and peaks at 50 years old [1]. Total knee arthroplasty (TKA) is currently proved to be an effective surgical method for patients with moderate or severe staged knee osteoarthritis and clinical practice has shown that it can significantly relieve the pain, improve the range of motion, increase the quality of life [2, 3]. However, adverse events after TKA remain a severe challenge for surgeons,Postoperative complications such as venous thromboembolism (VTE), infection, pyrophosphate fracture and dislocation have occurred in some patients [4, 5]. A study recommended that use of arthroscopic debridement is a feasible method for osteoarthritis [6]. This method only been shown to delay the time of TKA.Most of them need TKA to achieve the goal of treatment in the future. Several studies have evaluated the influence of TKA in patients with prior knee arthroscopy in recent years, but the results remain controversial[7-10]. Some studies suggested that prior arthroscopy resulted in longer operative time, higher rates of postoperative complications and reoperation, as well as a shorter implant working life. Other studies argued that prior arthroscopy has a negative impact on subsequent TKA [9].

In general, we still determine whether previous arthroscopy procedures can influence the clinical effects of subsequent future TKA and whether the surgeon and patients should pay more attention to previous procedures. Mutual controls were adopted by most previous studies to eliminate the impact of 
demographic variables-age, sex, body mass index between control groups and study groups. Nevertheless, some factors are hard to control and quantify, such as lifestyle, conditions of limbs exercise and rehabilitation. In this study, we selected the same patient to improve the study design. From September 2012 to July 2020, 56 patients fit the criteria for bilateral knee arthroplasty following knee arthroscopy. The clinical curative effects evaluation and the rate of complications were compared in both knees to evaluate the influence of prior arthroscopy on following TKA.

\section{Methods}

\section{Inclusion and exclusion criteria}

The inclusion criteria were: 1) patients who underwent simultaneously or staged TKA from September 2012 to July 2020. 2) patients with a history of unilateral arthroscopy . 3) patients with complete followup data.

The exclusion criteria were: 1) patients with prior a cruciate ligament reconstruction history. 2) patients who underwent knee revision. 3) patients who underwent TKA combined further operations under single anesthesia. 4) patients with infectious osteoarthritis, knee intramuscular fracture or other knee operation.

\section{General information}

From September 2012 to July 2020, 483 patients underwent TKA following arthroscopy in our hospital. Patients who underwent unilateral knee arthroplasty or patients with a history of bilateral knee arthroscopy were excluded, and finally, 56 patients (112 knees) were composite inclusion criteria. Among the 56 patients, 13 patients underwent simultaneous TKA and the other patients underwent staged TKA --7 patients were performed staged TKA within 1 month, 5 patients were more than 1 months but less than 12 months, and others were more than 12 months. We defined the knee with a prior arthroscopy history as group A and the other side as group B. All patients were followed up to 1 September 2020.

\section{Therapeutic process}

All the operations were performed by experienced surgeons in our hospital. Both knees were also completed by the same surgeon. The medial parapatellar approach was used in all patients. 30 patients were implanted with MP prosthesis while 26 patients were implanted with PS prosthesis, all the patients did not give receive a replacement of patella, but we rubbed abraded it down to gain a better motion curve. Negative pressure drainage tubes were placed on each side knee in every patient after the operation, and all drainage tubes were removed within 24 hours. Half an hour before the operation, the intravenous of traumatic acid (TXA) was chosen to prevent bleeding. We selected third-generation antibiotic prophylaxis to prevent infection and low molecular weight heparin as an anti-coagulent. When the drainage tubes were removed, all patients received daily rehabilitation training with the help of a professional therapist.

\section{Assessment}


The database that contains essential information laboratory examination imaging examination information of surgery and clinical records was obtained from electronic medical record systems in our hospital. Indexes were assessed with postoperative local complications and functional scores. Local complications include infection, pathological dislocation, periprosthetic fracture, aseptic loosening, ankylosis and wound complication. Functional evaluations included range of motion (ROM) Knee Society score and FJS score. Besides, visual analogue scale (VAS) was used for patient satisfaction. Comparisons of the aforesaid indexes between Group A and Group B were performed to evaluate the influence of the prior arthroscopy history on TKA.

\section{Statistical analysis}

All statistical testing methods used were performed using SPSS 25 software in this study. As for continuous variables (ROM/KSS/FJS), the Kolmogorov-Smirnov test was first applied to test normality. The data are reported using descriptive statistics such as mean \pm standard deviation or median \pm quartile interval for continuous variables or frequencies for categorical variables. Statistical comparisons of preoperative and postoperative ROM/KSS/FJS were completed with the paired t-test or Wilcoxon Pearson rank-sum test as well as measurement data between group $A$ and group $B$, and the chi-square test was applied to compare the rate of reoperation and complication between two groups. For all statistical comparisons, $\mathrm{P}<0.05$ was considered significant.

\section{Results}

56 patients met the inclusion criteria, including 7 males and 49 females. The mean age at the time of TKA was $62.89 \pm 6.30$ years (46-73) in the study group and 63.06 \pm 6.27 years (47-74) in the control group.The mean age, mean body mass index and ASA had no statistical significance between both groups.

The mean follow-up time of the study group was slightly longer than that of the control group (43 months vs 41 months), but there was no statistical significance. The mean follow-up time ranged from 26 to 77 months. All basic information is presented in Table 1.

A comparison of preoperative and postoperative KSS score between groups are shown in Table 2, including clinical score and functional score. There was no statistical significance in postoperative Knee Society clinical score and functional score between group $A$ and group $B(P>0.05)$.

ROM, FJS and VAS scores are shown in Table 3. There was no statistical significance in the preoperative ROM score, and the postoperative ROM score was also equivalent between groups (107.92 $\pm 10.98 \mathrm{ml}$ versus $108.75 \pm 10.98 \mathrm{ml}, \mathrm{P}=0.638$ ) at the latest follow-up. Similarly, the statistical analysis of FJS and VAS in both study groups showed no statistical significance.

During the follow-up period, we identified two local complications (3.6\%) in the study group, including one infection and one poor healing of the incision. We found that there were three complications $(5.4 \%)$ in the 
control group, including two infections and one poor healing of the incision. None of the patients experienced aseptic loosening, dislocation, pyrophosphate fracture or manipulations under anesthesia for stiffness. Although group B having a slightly higher rate of post complications, there was no statistical significance in two groups ( $5.4 \%$ versus $3.6 \%, P>0.05)$. The complications are shown in Table 4.

Three patients had revision surgery because of infection, one in Group A and the others in Group B. One patient in group $A$ required revision surgery at four months postoperatively with the culture results indicating Staphylococcus Aureus growth. The two patients in group B required the operation, one at eight months postoperatively and one at twenty-four months after operation. One culture result indicating Staphylococcus Aureus growth and the other culture results indicating staphylococcus epidermis growth. The rate of revision in Group A was slightly higher than Group B, but it was not statistically significant between the two groups ( $5.6 \%$ versus $2.8 \%, P>0.05)$.

\section{Discussion}

Only a few studies have paid attention to the influence of prior knee operation on subsequent TKA, including arthroscopy [7-10], ACL reconstruction [11, 12], non-ligamentous arthroscopic procedures and knee intramuscular fracture $[13,14]$, but the outcomes were conflicted. In this study, we compared bilateral knees in the same patient, when it comes to the local complication rates and clinical curative effects evaluation (FJS, KSS, ROM, VAS), we hold that there was no statistical significance. To our knowledge, it is the first comparative study in the same patient to examine the influence of prior arthroscopy on subsequent TKA.

Among these prior studies, only 4 previous studies evaluated the influence of prior arthroscopy on subsequent TKA [7-10].In 2009, Piedade [6] reported the influence on TKA following prior arthroscopy for the first time. In their study, 60 patients who underwent TKA with previous arthroscopic history were included as a study group and 1119 patients without knee surgery as a control group. Statistical analysis revealed that the study group had a higher postoperative complication rate and a lower working rate of the prosthesis. However, statistical analysis did not reveal a statistical significance in postoperative function and pain scores between two groups.

Then Werner and Barton $[8,10]$ studied the influence of the interval time between arthroscopy history and following TKA. Werner divided 3051 patients with previous knee arthroscopy into three separate cohorts according to the interval time: TKA within 6 months after knee arthroscopy $(n=681)$, TKA between 6 months and 1 year after knee arthroscopy $(n=1301)$ and TKA from 1 to 2 years after knee arthroscopy $(n=1069) .37235$ TKA patients without previous knee arthroscopy were created as the control group. The authors found the incidences of infection (OR 2.0, $P=0.004)$, stiffness (OR 2.0, $P=0.001$ ) and VTE (OR $1.6, P=0.047$ ) were higher in patients who underwent TKA within 6 months after knee arthroscopy compared to the patients in the control group. They also found that there was no increase in complications when TKA was performed more than six months after knee arthroscopy. 
Similarly, Barton found that the interval time was a crucial factor for the function of patients who performed TKA with a prior arthroscopy history. Patients who performed TKA within six months of prior arthroscopy had a significant reduction in OKS. However, Anthony [8] conducted the study for a long term with average follow-up time up to 9 years, and found there was no statistical significance in KSS, ROM, complication rates and the working time of prosthesis between both groups.

However, the above studies were all mutual control experiments from one patient to another patient. Although confounding variables, such as general condition operative procedures, and rehabilitation during hospitalization, were mitigated through the matched cohort, the impact of postoperative factors, such as lifestyle medication were hard to control. Compared to these studies, the strength of this particular study is self-controlled design. Patients who underwent simultaneously or staged TKA with a history of arthroscopy in one knee were included and the outcomes were compared in bilateral knees within the same patient.However, TKA performed by different surgical teams, using different prostheses or other different surgical techniques, were excluded. Therefore, this study can not only eliminate the discrepancy involving baseline, operative procedures, rehabilitation during hospitalization but also eliminate the impact of the postoperative lifestyle, the work conditions, use of medication and exercise conditions. This research method has already been applied to compare AS prosthesis and PS prosthesis [15], ComputerNavigated TKA and Conventional TKA [16], highly cross-linked prosthesis and conventional prosthesis [17], UKA and TKA [18, 19]. However, to our knowledge, it has not been adopted to evaluate the influence of prior arthroscopy on subsequent TKA.

At the latest follow-up, this study showed no statistical significance for KSS, FJS, ROM and VAS between two groups to previous reports. That is to say,the prior arthroscopy does not influence functional score and patient's satisfaction. Anthony [9] identified 480 patients (160 patients as arthroscopy group, 320 patients matched 2:1 as controls) and found no statistical significance in KSS score and ROM between two groups for ten years of follow-up. Piedade [7] identified 60 primary TKA with previous arthroscopic debridement as a study group and 1119 primary TKA without surgery as a control group. Statistical analysis of postoperative IKS and ROM showed no difference.

In our study, the complication rate was found to be equivalent between the two groups, which are consistent with most previous reports. In the 2:1 matched control study, Anthony [9] found the curatorship free of complication at 5 years and the survivorships free of revision were similar in both groups. Concerning the interval time between arthroscopy and TKA, Anthony found patients who had a knee arthroscopy within 1 year to receive TKA were not having a higher risk of complications or reoperations. Werner and Barton et al $[8,10]$ also reported there were not increasing in complications when TKA patient had an arthroscopy for more than six months, but patients undergoing TKA within six months of arthroscopy had a significantly higher rate of complications and reoperation. In contrast, Piedade [7] reported a higher postoperative complication rate in the arthroscopy group with a mean interval of 53 months. 
There are also limitations to our study. Foremost, this is a single-center clinical trial containing only 36 patients. Analysis of the impact of interval time between arthroscopy and TKA was not performed.Some documents have shown that the interval time between arthroscopy and TKA is a potential factor which may influence complication rates and clinical outcomes. Moreover, our study has the common shortcomings, in any retrospective cohort study, of including the possibility of selection or observational bias. Despite these limitations, we pioneered to conduct this comparison on the same patient, the results we have given are still credible.

\section{Conclusions}

For patients who have previously received arthroscopic knee surgery, it is safe to perform TKA, if the illness condition requires it, and the postoperative complications and recovery of knee function risks are the same as in common patients.

\section{Abbreviations}

TKA, total knee total knee arthroplasty

$\mathrm{ROM}$, range of motion

FJS, finger joint size

VAS,visual analogue scale

VTE, venous thromboembolism

$\mathrm{BMI}$, body mass index

ASA, american society of anesthesiologists

KSS, keen society score

UKA, unicompartmental knee arthroplasty

\section{Declarations}

\section{Ethics approval and consent to participate}

This study was approved by the Institutional Review Board of the affiliated hospital of Qingdao University. Written informed consent was obtained from all patients. No children (under 16 years old) were included in this study.

\section{Consent for publication}


Not applicable.

\section{Availability of data and materials}

The datasets generated during and/or analyzed during the current study are available from the corresponding author on reasonable request.

\section{Competing interests}

We declare that we have no Conflict of Interest.

\section{Funding}

We received no external funding for this study.

\section{Authors' contributions}

Authors Kui-shuai XU and Rui SHEN designed the study; Liang ZHANG,Tian-yu LI and Cai-lin Wang analyzed the data; Kui-shuai XUヌRui SHEN and Liang ZHANG wrote the manuscript; and Xia ZHAO and Teng-bo YU supervised the study.

\section{Acknowledgements}

We would like to thank all the staff in Department of Orthopaedic, Affiliated Hospital of Qingdao University for their contribution on our research.

\section{References}

1. Cross, M., et al., The global burden of hip and knee osteoarthritis: estimates from the global burden of disease 2010 study. Ann Rheum Dis, 2014. 73(7): p. 1323-30.

2. Ferket, B.S., et al., Impact of total knee replacement practice: cost effectiveness analysis of data from the Osteoarthritis Initiative. BMJ, 2017. 356: p. j1131.

3. Mathijssen, N.M.C., et al., Patient reported outcomes and implant survivorship after Total knee arthroplasty with the persona knee implant system: two year follow up. BMC Musculoskelet Disord, 2019. 20(1): p. 97.

4. Skou, S.T., et al., A Randomized, Controlled Trial of Total Knee Replacement. N Engl J Med, 2015. 373(17): p. 1597-606.

5. Thompson, R., et al., Arthrofibrosis After Total Knee Arthroplasty: Pathophysiology, Diagnosis, and Management. Orthop Clin North Am, 2019. 50(3): p. 269-279.

6. Spahn G, Kahl E, Muckley T, Hofmann GO, Klinger HM. 2008. Arthroscopic knee chondroplasty using a bipolar radiofrequency-based device compared to mechanical shaver: results of a prospective, randomized, controlled study. Knee Surg Sports Traumatol Arthrosc 16:565-73 
7. Piedade, S.R., et al., Is previous knee arthroscopy related to worse results in primary total knee arthroplasty? Knee Surg Sports Traumatol Arthrosc, 2009. 17(4): p. 328-33.

8. Werner, B.C., et al., Total Knee Arthroplasty Within Six Months After Knee Arthroscopy Is Associated With Increased Postoperative Complications. J Arthroplasty, 2015. 30(8): p. 1313-6.

9. Viste, A., et al., Prior Knee Arthroscopy Does Not Influence Long-Term Total Knee Arthroplasty Outcomes and Survivorship. J Arthroplasty, 2017. 32(12): p. 3626-3631.

10. Barton, S.B., G.J. McLauchlan, and S.J. Canty, The incidence and impact of arthroscopy in the year prior to total knee arthroplasty. Knee, 2017. 24(2): p. 396-401.

11. Watters, T.S., et al., Total Knee Arthroplasty After Anterior Cruciate Ligament Reconstruction: Not Just a Routine Primary Arthroplasty. J Bone Joint Surg Am, 2017. 99(3): p. 185-189.

12. Lizaur-Utrilla, A., et al., Total Knee Arthroplasty in Patients With Prior Anterior Cruciate Ligament Reconstruction. J Arthroplasty, 2018. 33(7): p. 2141-2145.

13. Piedade, S.R., et al., TKA outcomes after prior bone and soft tissue knee surgery. Knee Surg Sports Traumatol Arthrosc, 2013. 21(12): p. 2737-43.

14. Yoon, J.R., et al., Risk of Revision Following Total Knee Arthroplasty or High Tibial Osteotomy: A Nationwide Propensity-Score-Matched Study. J Bone Joint Surg Am, 2019. 101(9): p. 771-778.

15. SW, J., et al., Comparison of Anterior-Stabilized and Posterior-Stabilized Total Knee Arthroplasty in the Same Patients: A Prospective Randomized Study. 2019. 34(8): p. 1682-1689.

16. YH, K., et al., The Clinical Outcome of Computer-Navigated Compared with Conventional Knee Arthroplasty in the Same Patients: A Prospective, Randomized, Double-Blind, Long-Term Study. 2017. 99(12): p. 989-996.

17. YH, K., P.J.J.T.J.o. bone, and j.s.A. volume, Comparison of highly cross-linked and conventional polyethylene in posterior cruciate-substituting total knee arthroplasty in the same patients. 2014. 96(21): p. 1807-13.

18. CR, C., et al., Unicompartmental and total knee arthroplasty in the same patient. 2011. 24(4): p. 2738.

19. DF, D., et al., Unicompartmental knee arthroplasty compares favorably to total knee arthroplasty in the same patient. 2009. 32(4): p. undefined.

\section{Tables}

Table 1: Demographics and comorbidity profile of the patients 


\begin{tabular}{|c|c|c|c|}
\hline & Experimental group & Control group & $P$ \\
\hline Age(years) & $62.89 \pm 6.30 \rrbracket 46-73 \rrbracket$ & $63.06 \pm 6.27 \llbracket 47-74 \rrbracket$ & 0.160 \\
\hline Gender $\bigotimes$ male/female $\rrbracket$ & $7 / 49$ & $7 / 49$ & - \\
\hline \multirow[t]{2}{*}{ BMI $\triangle \mathrm{kg} / \mathbb{\text { 欧 }}$} & $27.96 \pm 2.97$ & $27.79 \pm 3.01$ & 0.541 \\
\hline & $\otimes 21.48-35.54 \rrbracket$ & $\rrbracket 22.03-35.54 \rrbracket$ & \\
\hline ASA & & & 0.813 \\
\hline 1 & 1 & 2 & \\
\hline 2 & 19 & 21 & \\
\hline 3 & 16 & 15 & \\
\hline Anesthesia & & & 0.810 \\
\hline General anesthesia & 31 & 32 & \\
\hline Non-general anesthesia & 25 & 24 & \\
\hline \multicolumn{4}{|l|}{ Comorbidity } \\
\hline Hypertension & 22 & 22 & 1.000 \\
\hline Coronary heart disease & 8 & 8 & 1.000 \\
\hline Diabetes & 14 & 14 & 1.000 \\
\hline Cerebral infarction & 1 & 1 & 1.000 \\
\hline Respiratory system & 6 & 6 & 1.000 \\
\hline Digestive system & 2 & 2 & 1.000 \\
\hline Urinary system & 1 & 1 & 1.000 \\
\hline
\end{tabular}

\section{Table 2ðClinical efficacy score}

\begin{tabular}{|lllllll|}
\hline & KS-C & & \multicolumn{3}{c|}{ KS-F } \\
& Pre-operation & Last follow up & P & Pre-operation & Last follow up & P \\
\hline Group A & $33.58 \pm 2.63$ & $83.58 \pm 6.54$ & 0.000 & $30.00 \pm 4.14$ & $81.53 \pm 6.19$ & 0.000 \\
\hline Group B & $34.19 \pm 2.93$ & $84.67 \pm 5.88$ & 0.000 & $30.28 \pm 3.15$ & $82.08 \pm 5.65$ & 0.000 \\
\hline P & 0.339 & 0.377 & & 0.757 & 0.657 & \\
\hline
\end{tabular}


Table 3囚ROM/FJS/VAS

\begin{tabular}{|llllll|}
\hline & ROM & & & FJS & VAS \\
\cline { 2 - 6 } & Pre- & Last & P & & \\
& operation & follow up & & & \\
Group A & $98.06 \pm 16.18$ & $107.92 \pm 10.98$ & 0.000 & $64.94 \pm 9.86$ & $7.47 \pm 1.34$ \\
Group B & $96.25 \pm 16.45$ & $108.75 \pm 10.98$ & 0.000 & $63.89 \pm 7.59$ & $7.58 \pm 1.20$ \\
\hline P & 0.378 & 0.638 & & 0.521 & 0.706 \\
\hline
\end{tabular}

Table 4囚Local complications

\begin{tabular}{|llll|}
\hline & Group A $(\mathrm{n}=56 \rrbracket$ & Group B\n=56囚 & $\mathrm{P}$ \\
\hline Complications & & & \\
\hline Infection & 1 & 2 & $\mathrm{P}>0.05$ \\
\hline Poor healing of the incisn & 1 & 1 & $\mathrm{P}>0.05$ \\
\hline Aseptic loosening & 0 & 0 & $\mathrm{P}>0.05$ \\
\hline Dislocation & 0 & 0 & $\mathrm{P}>0.05$ \\
\hline Periprosthetic fracturs & 0 & 0 & $\mathrm{P}>0.05$ \\
\hline Stiffness & 0 & 0 & $P>0.05$ \\
\hline Revision for any reason & 1 & 2 & $P>0.05$ \\
\hline
\end{tabular}

